\title{
Expression of hypoxia inducible factor-1a (HIF-1a) and glucose transporter-1 (GLUT-1) in ovarian adenocarcinomas: Difference in hypoxic status depending on histological character
}

\author{
MASANORI YASUDA ${ }^{1}$, MASAKI MIYAZAWA ${ }^{2}$, MARIKO FUJITA ${ }^{2}$, HIROSHI KAJIWARA ${ }^{2}$, TETSUJI IIDA ${ }^{3}$, \\ TAKESHI HIRASAWA ${ }^{3}$, TOSHINARI MURAMATSU ${ }^{3}$, MASARU MURAKAMI $^{3}$, MIKIO MIKAMI $^{3}$, \\ KAORU SAITOH $^{4}$, MICHIO SHIMIZU ${ }^{1}$, SUSUMU TAKEKOSHI ${ }^{2}$ and R. YOSHIYUKI OSAMURA ${ }^{2}$ \\ ${ }^{1}$ Department of Pathology, Saitama Medical University International Medical Center, Hidaka, Saitama; Departments of \\ ${ }^{2}$ Pathology, and ${ }^{3}$ Obstetrics and Gynecology, Tokai University School of Medicine, Bohseidai, Isehara, Kanagawa; \\ ${ }^{4}$ Department of Obstetrics and Gynecology, Saint Marianna Medical University, Kawasaki, Kanagawa, Japan
}

Received July 6, 2007; Accepted August 29, 2007

\begin{abstract}
The expression of hypoxia inducible factor- $1 \alpha$ (HIF-1 $\alpha$ ) and glucose transporter-1 (GLUT-1) was immunohistochemically analyzed in ovarian adenocarcinomas with the aim of elucidating whether hypoxic status is associated with histological type or structural character. The following ovarian adenocarcinomas were used: serous adenocarcinoma (SEA), 21 cases; mucinous adenocarcinoma (MUA), 19 cases; endometrioid adenocarcinoma (ENA), 16 cases; clear cell adenocarcinoma (CLA), 19 cases. High-level expression (3+) of HIF- $1 \alpha$ was observed in $100 \%$ of SEAs, $58 \%$ of MUAs, $100 \%$ of ENAs and $89 \%$ of CLAs, and high-level expression of GLUT-1 in $76 \%$ of SEAs, $26 \%$ of MUAs, $50 \%$ of ENAs and $67 \%$ of CLAs. Heterogeneous or localized staining was relatively evident for GLUT-1. Immunohistochemical profiles were in accord with the immunoblotting and mRNA levels of both markers. ELISA for the detection of active HIF-1 demonstrated that HIF-1 is strongly activated in SEAs, ENAs and CLAs as compared to MUAs. Our results show that GLUT-1 overexpression is to some extent regulated by HIF-1 $\alpha$ and is also strongly associated with histological features, i.e., papillary or stratified structure accompanied by little or no vascular stroma. In conclusion, hypoxic status differs according to the histological type of ovarian adenocarcinoma and the micro-environmental conditions of each type.
\end{abstract}

\section{Introduction}

Malignant tumors are considered to be in a more or less hypoxic state, with highly malignant tumors being especially hypoxia-resistant (1). Hypoxia inducible factor-1 (HIF-1),

Correspondence to: Dr Masanori Yasuda, Department of Pathology, Saitama Medical University International Medical Center, 1397-1 Yamane, Hidaka, Saitama 350-1298, Japan

E-mail: m_yasuda@saitama-med.ac.jp

Key words: ovary, adenocarcinoma, hypoxia, HIF-1 $\alpha$, GLUT-1 which is a heterodimer made up of an $\alpha$ and $\beta$ subunit, is one of the interesting recently discovered factors involved in tumor development and histogenesis (1). The heterodimer of HIF-1 in the nucleus is biologically functional when it is bound to hypoxia response elements (HREs). It is known that pathophysiological phenomena, including glucose transport, glycolysis, angiogenesis, erythropoiesis and the inhibition of apoptosis, are usually regulated by transcription factors such as HIF-1 $\alpha$ (2). In this regulation, the expression level of the HIF- $1 \alpha$ subunit is a determinant of HIF-1 transcription activity (2) and the loss of the von Hippel-Lindau tumor suppressor gene results in constitutive high-level expression of HIF- $1 \alpha$ (3). In general, when compared to non-malignant tumor cells or normal tissue cells the glucose utilization rate is increased in malignant tumor cells (4). One or more members of the glucose transporter (GLUT) gene family are expressed in all mammalian cells. GLUTs are integral membrane glycoproteins that play a key role in facilitating glucose transport (5). GLUT-1 is a representative member of the family and is widely distributed in normal tissues such as erythrocytes and endothelial cells at the blood-brain barrier (6-8). Various studies have shown a close relationship between GLUT-1 expression and carcinogenesis, tumor development or the unfavorable prognosis of various malignancies (9-12).

We have observed a progressive increase in HIF-1 $\alpha$ and GLUT-1 expression in the following order: ovarian serous and mucinous tumors from adenomas, borderline tumors, and finally adenocarcinomas (data not shown). These findings are basically the same as those of previous reports, differing only in part (13). In this study, we focused on the expression of HIF- $1 \alpha$ and GLUT-1 in ovarian adenocarcinomas and analyzed the relationship between hypoxic status and histological type or structure.

\section{Materials and methods}

Diseases (Table I). The 74 surgically treated ovarian carcinomas used comprised 21 cases of serous adenocarcinoma (SEA), 19 cases of mucinous adenocarcinoma (MUA), 16 
Table I. Ovarian adenocarcinomas examined.

\begin{tabular}{lr}
\hline Total patients & 74 \\
Age (years) & \\
$\quad$ Range & $30-81$ \\
Mean & 53.2 \\
Histology & \\
$\quad$ Serous adenocarcinoma & 21 \\
Mucinous adenocarcinoma & 19 \\
Endometrioid adenocarcinoma & 16 \\
Clear cell adenocarcinoma & 18 \\
Stage (FIGO) & Ia-IIIc \\
\hline
\end{tabular}

cases of endometrioid adenocarcinoma (ENA) and 18 cases of clear cell adenocarcinoma (CLA).

Immunohistochemistry. Formalin-fixed and paraffin-embedded tissue blocks were cut into $4 \mu \mathrm{m}$-thick sections for immunohistochemistry as well as for hematoxylin and eosin staining. The indirect peroxidase method was performed using heat antigen retrieval at $95^{\circ} \mathrm{C}$ and citrate buffer, $\mathrm{pH} 6.0$. The primary antibodies were as follows: HIF-1 $\alpha$, monoclonal, mouse, clone $\mathrm{H} 1 \alpha 67$, diluted 1:100 (Novus Biologicals, Littleton, CO) and GLUT-1, polyclonal, rabbit, diluted 1:50 (Dako, Carpinteria, CA). After incubation, sections were counterstained with hematoxylin. The extent of HIF-1 $\alpha$ and GLUT-1 expression was semi-quantitatively assessed according to the following scoring scheme: negative $(0 \%)$; weak, $1+(<10 \%)$; intermediate, $2+(10-50 \%)$; marked, $3+$ $(>50 \%)$. Preparations of cultured HeLa cells were used as positive controls for HIF-1 $\alpha$, and erythrocytes in each section were used as positive controls for GLUT-1. Nuclear and/or cytoplasmic expression of HIF-1 $\alpha$ staining was regarded as being positive. For GLUT-1, membrane-predominant staining was regarded as being positive. As negative controls, adjacent sections were incubated using non-immune mouse or rabbit serum instead of primary antibodies. Statistical analysis was performed using the $\chi^{2}$ test to determine whether there was a significant difference in the extent of immunohistochemical expression.

Immunoblotting. Using fresh materials from the representative cases of each histological type, total proteins were separated by electrophoresis on a $10 \%$ SDS-polyacrylamide gel and transferred to an immunobilon-P membrane (Millipore, Bedford, MA). The membrane was then blocked with 5\% non-fat milk in phosphate-buffered saline containing $0.5 \%$ Tween-20 (PBS-T) at room temperature for $1 \mathrm{~h}$, incubated overnight at $4{ }^{\circ} \mathrm{C}$ with anti-HIF- $1 \alpha$ and anti-GLUT- 1 antibodies and diluted 1:250 in 5\% non-fat milk in PBS-T. Horseradish peroxidase-conjugated anti-mouse complex for HIF- $1 \alpha$ and anti-rabbit complex for GLUT-1 were visualized using an ECL Plus Kit (Amersham Bioscience Corp., Piscataway, NJ). As positive controls, cultured HeLa cells and human red blood cells were used for HIF- $1 \alpha$ and GLUT-1, respectively.
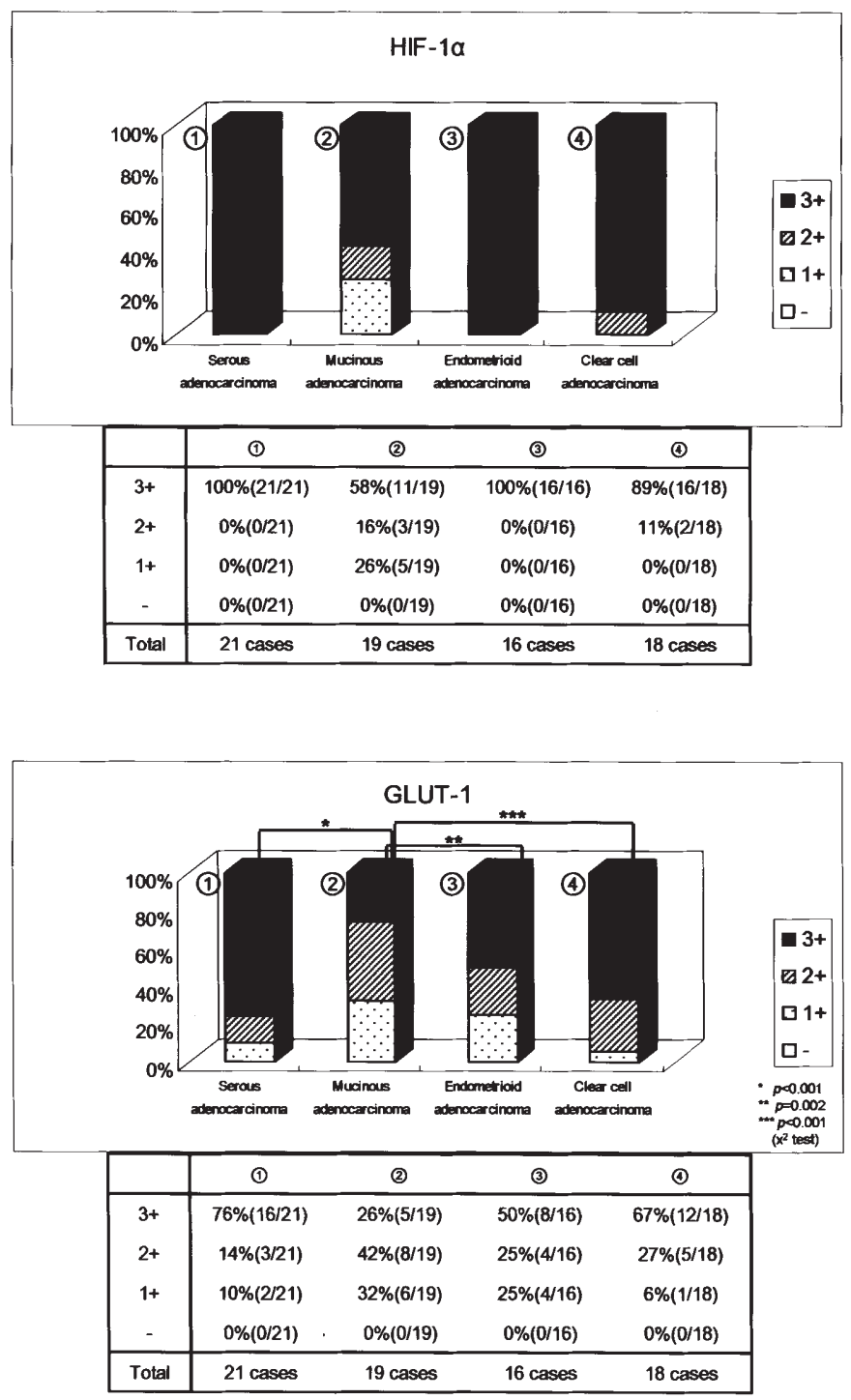

Figure 1. Comparison of immunohistochemical expression scores of HIF-1 $\alpha$ and GLUT-1. HIF- $1 \alpha$ and GLUT-1 expression in MUAs tended to be relatively weak compared to other types. The difference in GLUT-1 expression was statistically demonstrated by the $\chi^{2}$ test (HIF- $1 \alpha$, indeterminate).

Real-time $R T-P C R$. For a representative case of each histological type, mRNA expression levels of HIF-1 $\alpha$ and GLUT-1 were quantitatively assessed by real-time RT-PCR methods with the ABI PRISM 7700 System (TaqMan PCR, Applied Biosystems, Foster City, CA) using Assay-onDemand primers and probe sets $\mathrm{Hs} 00153153 \mathrm{ml}$ for HIF-1 $\alpha$ and Hs00197884ml for GLUT-1. Thermal cycling conditions consisted of $95^{\circ} \mathrm{C}$ for $10 \mathrm{~min}$ followed by 50 cycles of amplification at $95^{\circ} \mathrm{C}$ for $15 \mathrm{sec}$ and $60^{\circ} \mathrm{C}$ for $1 \mathrm{~min}$. Human glyceraldehyde-3-phosphate dehydrogenase (GAPDH) mRNA was measured as a control using Hs $99999905 \mathrm{ml}$. The relative expression of each mRNA was calculated by the $\Delta \mathrm{Ct}$ method.

ELISA. To investigate the activity of HIF-1, which is made up of HIF- $1 \alpha$ and HIF-1ß in the nucleus, a TransAM HIF-1 Kit was utilized according to the manufacturer's instructions (Active Motif, Carlsbad, CA). First, nuclear extracts produced from the fresh materials were put in the wells of a 96-well 


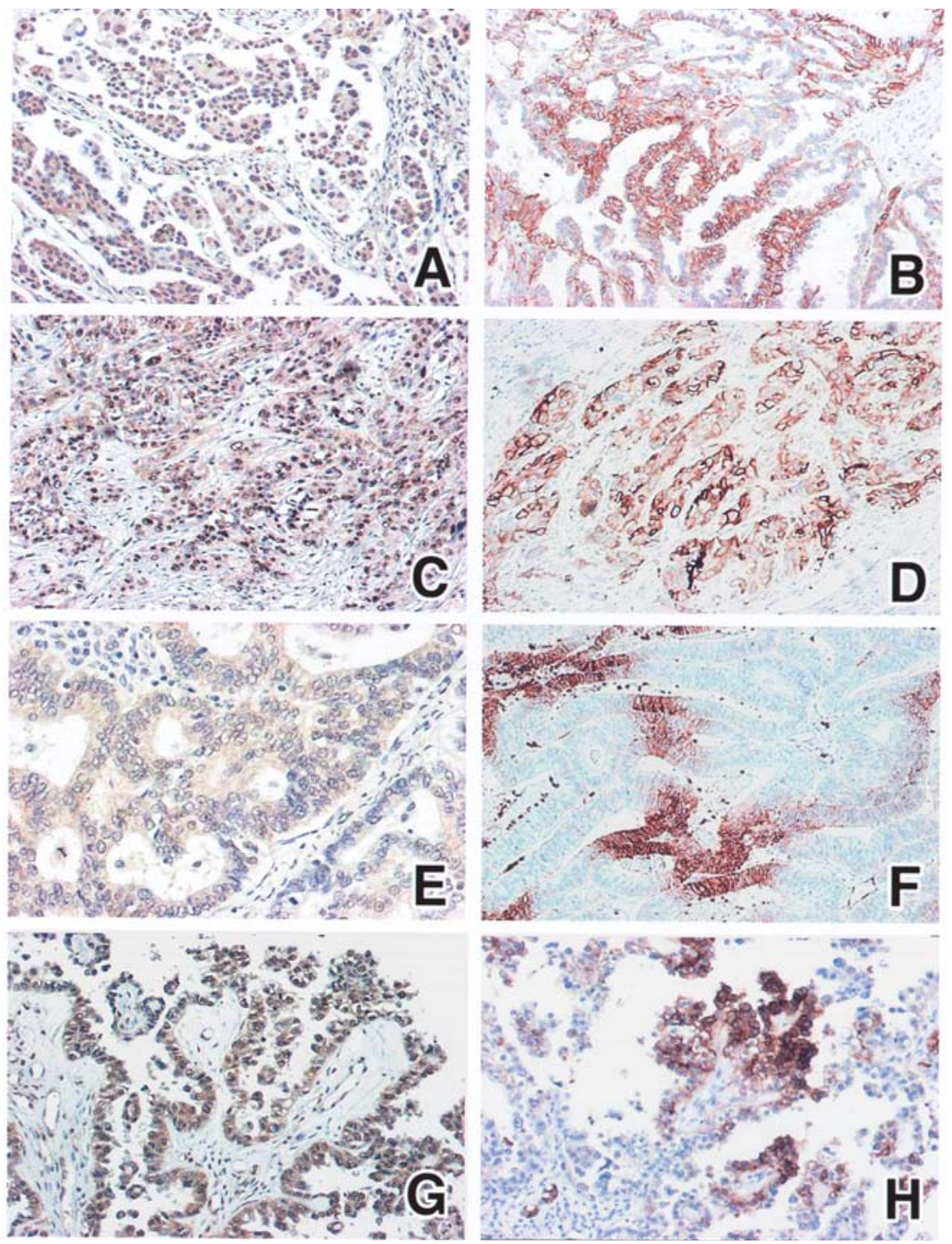

Figure 2. Immunohistochemical expression of HIF-1 $\alpha$ (A, C, E, G) and GLUT-1 (B, D, F, H). (A) SEA, invasive papillary and solid nests. (B) SEA, papillary and bridging tubular structure. (C) MUA, invasive distorted tubules. (D) MUA, separate tubular structure. (E) ENA, well-formed tubular nests. (F) ENA, fused tubular nests. (G) CLA, papillary protrusion with stroma. (H) CLA, piled-up carcinoma cells. Positive staining of HIF-1 $\alpha$ was observed diffusely and variably in both the nucleus and cytoplasm of carcinoma cells (A, C, G) or predominantly in the cytoplasm (E). The cell membrane was highlighted by GLUT-1 staining in a localized manner $(\mathrm{B}, \mathrm{D}, \mathrm{F}, \mathrm{H})$, and the positive reaction was also found to be strengthened around the top away from the stroma (B, H). SEA, serous adenocarcinoma; MUA, mucinous adenocarcinoma; ENA, endometrioid adenocarcinoma; CLA, clear cell adenocarcinoma.

plate with immobilized double-stranded DNA sequences containing the HIF-1 binding HREs. HIF-1 binding was detected using a primary mouse anti-HIF-1 antibody and a secondary horseradish peroxidase-labeled anti-mouse antibody. Absorbance was then measured spectrophotometrically at $450 \mathrm{~nm}$. The Kruskal-Wallis test was used to evaluate the differences in HIF-1 activity among the ovarian adenocarcinomas.

\section{Results}

Immunohistochemistry (Figs. 1 and 2)

$H I F-1 \alpha$. A positive reaction was most frequently observed in both the cytoplasm and nuclei of tumor cells. In general, the staining score (extent) paralleled staining intensity and nuclear labeling. A strong staining score $(3+)$ was observed in $100 \%(21 / 21)$ of SEAs, $58 \%$ (11/19) of MUAs, $100 \%(16 / 16)$ of ENAs and $89 \%(16 / 18)$ of CLAs. In contrast, weak (1+) or negative staining was observed in 26\% (5/19) of MUAs. No apparent positive reaction was observed in the stromal components.

GLUT-1. All of the examined adenocarcinomas showed various degrees of positive staining. The positive reaction was detected in both the cytoplasm and cell membrane. As the staining score increased, cell membrane staining tended to be more accentuated. Marked staining (3+) was observed in $76 \%$ $(16 / 21)$ of SEAs, $26 \%$ (5/19) of MUAs, $50 \%$ (8/16) of ENAs and $67 \%(12 / 18)$ of CLAs. The stromal components were basically negative.

Comparison of HIF-1 $\alpha$ and GLUT-1 expression. In most of the examined cases, HIF-1 $\alpha$ was more extensively and uniformly expressed than GLUT-1. However, GLUT-1 expression tended to be localized and to show a gradual enhancement with increasing distance from the vascular stroma. Statistically, 


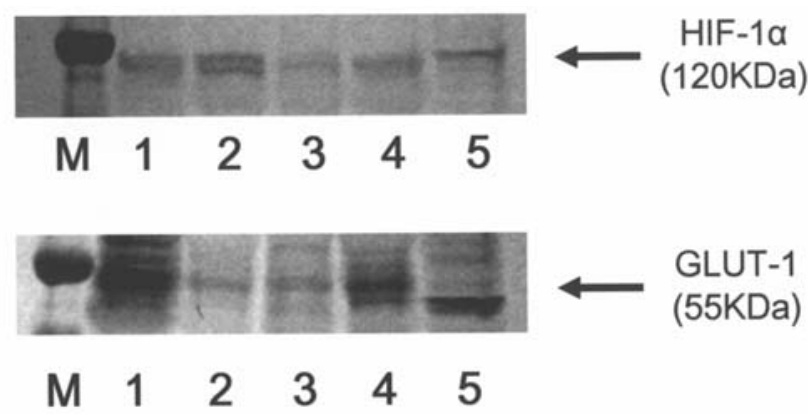

Figure 3. Immunoblotting analysis of HIF-1 $\alpha$ and GLUT-1. The positive reaction in each adenocarcinoma corresponded to immunohistochemical expression levels. M, marker; Lane 1, serous adenocarcinoma; Lane 2, mucinous adenocarcinoma; Lane 3, endometrioid adenocarcinoma; Lane 4, clear cell adenocarcinoma; Lane 5, positive control.
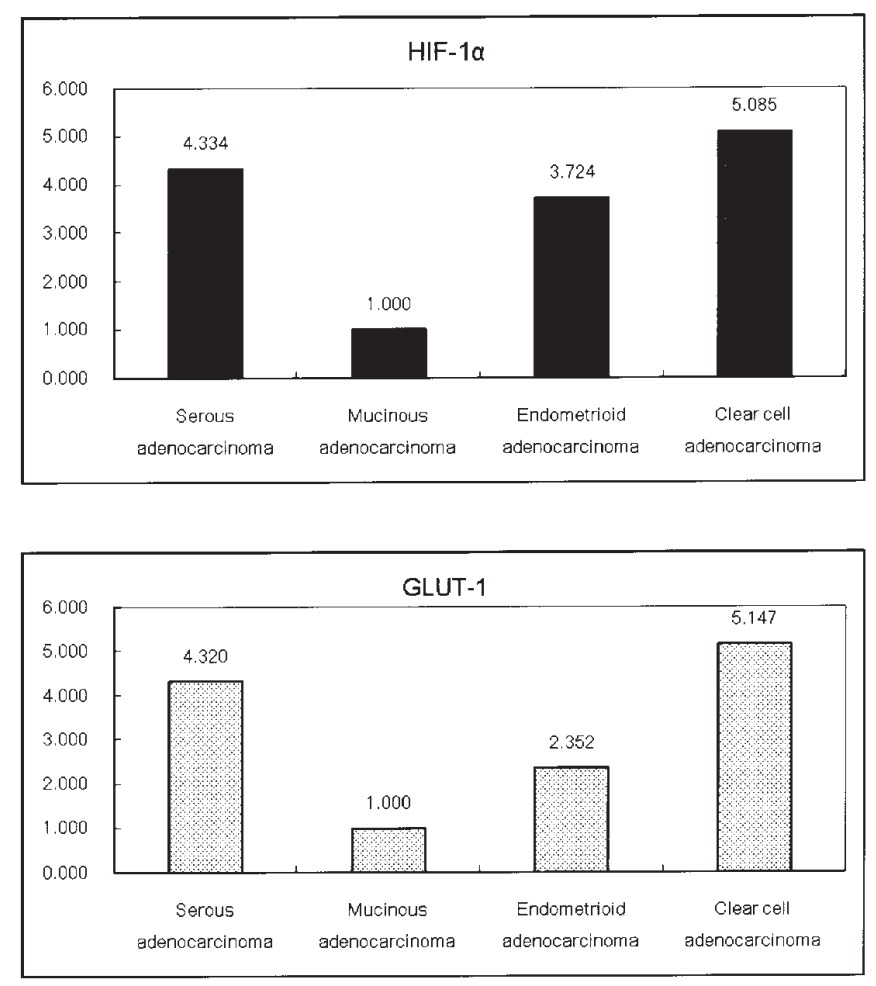

Figure 4. Relative mRNA levels of HIF-1 $\alpha$ and GLUT-1 by real-time RTPCR. Expression levels of mRNA differed considerably among ovarian adenocarcinomas, reflecting the immunohistochemical staining profiles.

significant difference in the staining score was demonstrated to exist between MUAs and SEAs $(p<0.001)$, ENAs $(p=0.002)$ and CLAs $(\mathrm{p}<0.001)$.

Immunoblotting (Fig. 3). The results of immunoblotting analysis of isolated protein were in accord with the HIF-1 $\alpha$ and GLUT-1 expression levels determined by immunohistochemical staining.

Real-time RT-PCR (Fig. 4). Quantitative analysis of HIF-1 $\alpha$ and GLUT-1 mRNA levels demonstrated that mRNA levels were in accord with the immunohistochemical expression profiles.

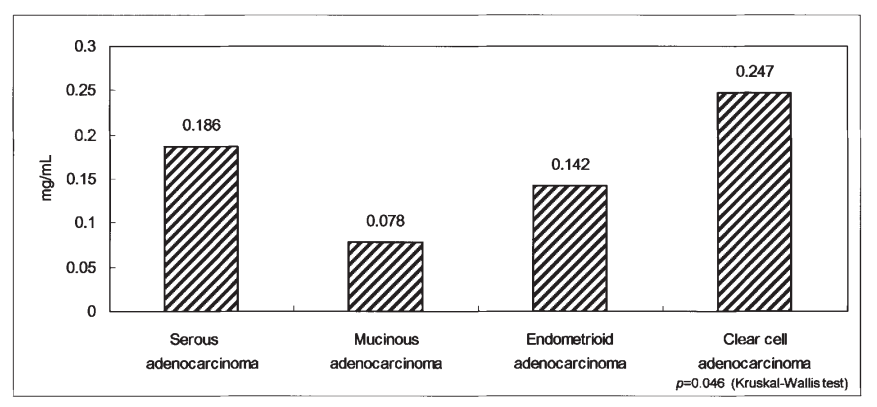

Figure 5. ELISA for the detection of HIF-1 activity in nuclei. It was found that the activity differences among ovarian adenocarcinomas corresponded to the differences in the immunohistochemical expression profiles, statistically demonstrated by the Kruskal-Wallis test.

ELISA (Fig. 5). Activation status reflected the immunohistochemical expression profiles. There were significant differences among the ovarian adenocarcinomas in HIF-1 activity, demonstrated by the Kruskal-Wallis test ( $\mathrm{p}=0.046)$.

\section{Discussion}

HIF- $1 \alpha$ is known to play an essential role in adapting the cellular environment to a hypoxic status by inducing the expression of various hypoxia response molecules, including GLUT-1 (1-3). Semenza reported that increased HIF-1 $\alpha$ is overexpressed as a result of intratumoral hypoxia, leading to treatment failure and a poor prognosis for the malignancies (2,14-19). Increased GLUT-1 expression has also been demonstrated in various malignant tumors (9-12,21-24). In colorectal cancer (23) and thyroid cancer (24), it has been reported that GLUT-1 overexpression is related to a higher frequency of lymph node metastasis and extra-organ growth. The present study focuses on the analysis of the hypoxic status in ovarian adenocarcinomas by looking at differences in histological type and structural characteristics.

The results suggest that GLUT-1 expression is considerably controlled by HIF- $1 \alpha$ expression, as most of the GLUT-1positive areas were contained within HIF-1 $\alpha$-positive areas. However, HIF-1 $\alpha$ expression was characterized by uniform staining while GLUT-1 overexpression was found to be heterogeneous and localized; adenocarcinoma cells far from stroma containing vascular vessels were clearly labeled (Figs. 1 and 2). GLUT-1 expression in ovarian adenocarcinomas may not be fully regulated by the key transcriptional regulator of HIF- $1 \alpha$. Heterogeneous GLUT-1 expression cannot be satisfactorily explained solely through regulation by HIF- $1 \alpha$. It is possible that GLUT-1 overexpression is more strongly affected by micro-environmental conditions than HIF- $1 \alpha$ expression. Interestingly, the discrepancy between HIF- $1 \alpha$ expression and GLUT-1 expression was most evident in ENAs. In many ENAs, the GLUT-1-positive area was considerably more narrow than the HIF- $1 \alpha$-positive area.

We recently reported that papillary carcinomas of the thyroid gland show marked GLUT-1 expression in comparison with follicular carcinomas (24). The difference in GLUT-1 expression between papillary carcinomas and follicular carcinomas is thought to depend on the manner of blood supply. We can therefore speculate that papillary proliferation 
would lead to the stimulation of GLUT-1 expression. For ovarian adenocarcinomas, papillary proliferation or the stratified structure of carcinoma cells accompanied by fewer vascular vessels could be responsible for the overexpression of GLUT-1 as well as HIF-1 $\alpha$ in SEAs and CLAs. GLUT-1 overexpression in carcinoma cells enclosing necrotic areas and in those farther away from vascular stroma is considered to reflect strong hypoxic status in SEAs and CLAs. The relatively weak expression of HIF-1 $\alpha$ and GLUT-1 in MUAs may be due to the following phenomena: many MUAs have a comparatively large amount of accompanying stroma intervening between the nests, and carcinoma cells are less stratified. Compared to large-sized nests, small-sized infiltrative nests with high proliferative potential of MUAs are more frequently positive for HIF-1 $\alpha$ and GLUT-1. As observed in follicular carcinomas of the thyroid gland, blood supply for carcinoma cells may to some extent be maintained in MUAs.

Cantuaria et al (11) concluded that GLUT-1 status is an independent prognostic factor of the response to chemotherapy in advanced ovarian carcinomas. It is reported that patients overexpressing GLUT-1 have a significantly lower diseasefree survival rate. In the multivariate analysis, only residual tumor and marked GLUT-1 expression maintained an independent prognostic value in regards to the response to chemotherapy (11). However, Birner et al reported that HIF-1 $\alpha$ overexpression alone has no impact on the prognosis of ovarian cancer (16). We are now working to analyze the association between the prognosis and the overexpression of both markers using a large-scale group of ovarian adenocarcinomas. In the present study at least, high expression of both HIF-1 $\alpha$ and GLUT-1 seemed to be closely related to the aggressive clinical outcome which leads, irrespective of chemotherapy, to short post-operative survival times.

To confirm the accuracy and significance of the immunostaining profiles, we performed immunoblotting analysis (Fig. 3), a fixed-quantity analysis of mRNA (Fig. 4) and ELISA for the detection of active HIF-1 $\alpha$ (Fig. 5). Isolated proteins detected in the immunoblotting analysis were observed to correspond to HIF-1 $\alpha$ and GLUT-1, respectively. The difference in immunostaining profiles was considered to be related to the mRNA levels of HIF- $1 \alpha$ and GLUT-1, which were higher in SEAs and CLAs but lower in MUAs. The results suggest that the mRNA levels of both markers closely control immunohistochemical expression patterns. It was also found that the immunostaining profiles closely reflected the HIF-1 activity detected by ELISA. We therefore believe that immunostaining in the nucleus is a reliable hallmark indicating whether HIF-1 is functionally activated.

The suppression of GLUT-1 mRNA is expected to inhibit tumor growth, as shown experimentally by the transfection of antisense mRNA into tumor cells (25). A unique strategy, which involves the administration of certain cytotoxic agents conjugated to glucose, has been proposed to treat ovarian malignancies with accentuated glucose uptake (26). HIF-1 $\alpha$ suppression has also been achieved through the administration of a certain agent in vitro (27). Our experimental study, using cultured ovarian clear cell carcinoma, suggests that HIF-1 $\alpha$ could be suppressed by rapamycin (data not shown).

In conclusion, HIF- $1 \alpha$ and GLUT- 1 expression tend to be associated, but with significant differences between them.
GLUT-1 expression was strongest in ovarian adenocarcinoma cells with papillary-structural components characterized by SEAs and CLAs. Our study raises the possibility that, in ovarian adenocarcinomas with marked expression of HIF- $1 \alpha$ and GLUT-1, both markers could be therapeutic targets in addition to conventional chemotherapies.

\section{References}

1. Zhong H, De Marzo AM, Laughner E, Lim M, Hilton DA, Zagzag D, Buechler P, Isaacs WB, Semenza GL and Simons JW: Overexpression of hypoxia-inducible factor 1alpha in common human cancers and their metastases. Cancer Res 59: 5830-5835, 1999.

2. Semenza GL: HIF-1 and tumor progression: pathophysiology and therapeutics. Trends Mol Med 8: 62-67, 2002.

3. Huang LE, Gu J, Scheau M and Bunn HF: Regulation of hypoxia-inducible factor 1 alpha is mediated by an $\mathrm{O}_{2}$-dependent degradation domain via the ubiquitine-proteasome pathway. Proc Natl Acad Sci USA 95: 7987-7992, 1998.

4. Younes M, Lechago LV and Lechago J: Overexpression of the human erythrocyte glucose transporter occurs as a late event in human colorectal carcinogenesis and is associated with an increased incidence of lymph node metastases. Clin Cancer Res 2: 1151-1154, 1996.

5. Wheeler TJ and Hinkle PC: The glucose transporter of mammalian cells. Annu Rev Physiol 47: 503-517, 1985.

6. Froehner SC, Davies A, Baldwin SA and Lienhard GE: The blood-nerve barrier is rich in glucose transporter. J Neurocytol 17: 173-178, 1988.

7. Pardridge WM, Boado RJ and Farrell CR: Brain-type glucose transporter (GLUT-1) is selectively localized to the blood-brain barrier. Studies with quantitative Western blotting and in situ hybridization. J Biol Chem 265: 18035-18040, 1990.

8. Agus DB, Gambhir SS, Pardridge WM, Spielholz C, Baselga J, Vera JC and Golde DW: Vitamin C crosses the blood-brain barrier in the oxidized from through the glucose transporters. J Clin Invest 100: 2842-2848, 1997.

9. Haber RS, Rathan A, Weiser KR, Pritsker A, Itzkowitz SH, Bodian C, Slater G, Weiss A and Burstein DE: GLUT1 glucose transporter expression in colorectal carcinoma: a marker for poor prognosis. Cancer 83: 34-40, 1998

10. Ogawa J, Inoue H and Koide S: Glucose-transporter-type-I-gene amplification correlates with sialyl-Lewis-X synthesis and proliferation in lung cancer. Int J Cancer 74: 189-192, 1997.

11. Cantuaria G, Fagotti A, Ferrandina G, Magalhaes A, Nadgi M, Angioli R, Penalver M, Mancuso S and Scambia G: GLUT-1 expression in ovarian carcinoma: association with survival and response to chemotherapy. Cancer 92: 1144-1150, 2001.

12. Kuwamura T, Kusakabe T, Sugino T, Watanabe K, Fukuda T, Nashimoto A, Honma K and Suzuki T: Expression of glucose transporter-1 in human gastric carcinoma: association with tumor aggressiveness, metastasis, and patient survival. Cancer 92: 634-641, 2001.

13. Kalir T, Wang BY, Goldfischer M, Haber RS, Reder I, Demopoulos R, Cohen CJ and Burstein DE: Immunohistochemistry staining of GLUT1 in benign, borderline, and malignant ovarian epithelia. Cancer 94: 1078-1082, 2002.

14. Koukourakis MI, Giatromanolaki A, Skarlatos J, Corti L, Blandamura S, Piazza M, Gatter KC and Harris AL: Hypoxia inducible factor (HIF- $1 \alpha$ and HIF-2 $\alpha$ ) expression in early esophageal cancer and response to photodynamic therapy and radiotherapy. Cancer Res 61: 1830-1832, 2001

15. Aebersold DM, Burri P, Beer KT, Laissue J, Djonov V, Greiner RH and Semenza GL: Expression of hypoxia-inducible factor-1alpha: a novel predictive and prognostic parameter in the radiotherapy of oropharyngeal cancer. Cancer Res 61: 2911-2916, 2001.

16. Birner P, Schindl M, Obermair A, Breitenecker G and Oberhuber G: Expression of hypoxia-inducible factor 1alpha in epithelial ovarian tumors: its impact on prognosis and on response to chemotherapy. Clin Cancer Res 7: 1661-1668, 2001

17. Birner P, Schindl M, Obermair A, Plank C, Breitenecker G and Oberhuber G: Overexpression of hypoxia-inducible factor 1alpha is a marker for an unfavorable prognosis in early-stage invasive cervical cancer. Cancer Res 60: 4693-4696, 2000. 
18. Bos R, Zhong H, Hanrahan CF, Mommers EC, Semenza GL, Pinedo HM, Abeloff MD, Simons JW, van Diest PJ and van der Wall E: Levels of hypoxia-inducible factor-1 alpha during breast carcinogenesis. J Natl Cancer Inst 93: 309-314, 2001.

19. Zagzag D, Zhong H, Scalzitti JM, Laughner E, Simons JW and Semenza GL: Expression of hypoxia-inducible factor 1alpha in brain tumors: association with angiogenesis, invasion, and progression. Cancer 88: 2606-2018, 2000.

20. Ikeda E: Cellular response to tissue hypoxia and its involvement in disease progression. Pathol Int 55: 603-610, 2005.

21. Wang BY, Kalir T, Sabo E, Sherman DE, Cohen C and Burstein DE: Immunohistochemical staining of GLUT1 in benign, hyperplastic, and malignant endometrial epithelia. Cancer 88: 2774-2781, 2000.

22. Haber RS, Weiser KR, Pritsker A, Reder I and Burstein DE: GLUT1 glucose transporter expression in benign and malignant thyroid nodules. Thyroid 7: 363-367, 1997.

23. Younes M, Lechago LV and Lechago J: Overexpression of the human erythrocyte glucose transporter occurs as a late event in human colorectal carcinogenesis and is associated with an increased incidence of lymph node metastases. Clin Cancer Res 2: 1151-1154, 1996
24. Yasuda M, Ogane N, Hayashi H, Kameda Y, Miyagi Y, Iida T, Mori Y, Tsukinoki K, Minematsu T and Osamura Y: Glucose transporter-1 expression in the thyroid gland: clinicopathological significance for papillary carcinoma. Oncol Rep 14: 1499-1504, 2005.

25. Noguchi Y, Saito A, Miyagi Y, Yamanaka S, Marat D, Doi C, Yoshikawa T, Tsuburaya A, Ito T and Satoh S: Supression of facilitative glucose transporter $1 \mathrm{mRNA}$ can suppress tumor growth. Cancer Lett 154: 175-182, 2000.

26. Cantuaria G, Magalhaes A, Penalver M, Angioli R, Braunschweiger P, Gomez-Marin O, Kanhoush R, GomezFernandez C and Nadji M: Expression of GLUT-1 glucose transporter in borderline and malignant epithelial tumors of the ovary. Gynecol Oncol 79: 33-37, 2000.

27. Hudson CC, Liu M, Chiang GG, Otterness DM, Loomis DC, Kaper F, Giaccia AJ and Abraham RT: Regulation of hypoxiainducible factor 1alpha expression and function by mammalian target of rapamycin. Mol Cell Biol 22: 7004-7014, 2002. 